\title{
Semen Ziziphi Spinosae and Fructus Gardeniae extracts synergistically improve learning and memory of a mouse model
}

\author{
BAOLI LI ${ }^{1 *}$, ZHAOYING FU $^{2 *}$, RUI HU $^{3 *}$, YAHUI CHEN ${ }^{4}$ and ZHENGXIANG ZHANG ${ }^{1}$ \\ Departments of ${ }^{1}$ Pharmacology and ${ }^{2}$ Molecular Biology, Medical College of Yan'an University, Yan'an 716000; \\ ${ }^{3}$ Department of Pharmacology, Shanxi University of Traditional Chinese Medicine, Xi'an 712046; \\ ${ }^{4}$ Department of Physiology, Medical College of Yan'an University, Yan'an 716000, P.R. China
}

Received July 19, 2012; Accepted October 18, 2012

DOI: 10.3892/br.2012.38

\begin{abstract}
Semen Ziziphi Spinosae (SZS) and Fructus Gardeniae (FG) are two herbs commonly used in traditional Chinese medicine. Previous studies have suggested that Fructus Gardeniae as well as Semen Ziziphi Spinosae are able to regulate the function of the central nervous system. However, their effect on learning and memory has yet to be elucidated. In this study, we examined the effect of SZS and FG on the learning and memory of mice using the methods of step-through and -down passive avoidance tasks and Morris water maze tasks. The results showed that SZS and FG extracts have certain effects on improving the performance of the learning and memory-impaired mouse model. Of note, compound extracts of SZS and FG have a synergistic effect on the learning and memory of mice.
\end{abstract}

\section{Introduction}

Semen Ziziphi Spinosae (SZS) and Fructus Gardeniae (FG) are two Chinese herbal medicines frequently used in traditional Chinese medicine (TCM). SZS is the dry ripe seed of the deciduous shrub or small shrub Ziziphus jujuba Mill. var. spinosa (Bunge) Hu ex H.F. Chou. FG is the dry ripe fruit of the evergreen shrub Gardenia jasminoides Ellis $(1,2)$, usually used as an adjuvant medicine although it could also be used independently (3).

Extracts obtained from SZS have been reported to have therapeutic effects on insomnia, sleep disorders, anxiety and neurasthenia. Our previous study showed that oil extracts obtained from FG have the function of sedation and hypnosis (6). Thus, SZS as well as FG are suggested to have the function of regulating the central nervous system. Our

Correspondence to: Dr Baoli Li, Department of Pharmacology, Medical College of Yan'an University, Yan'an, 716000, P.R. China E-mail: 1bl_0812@hotmail.com

*Contributed equally

Key words: learning and memory, Ziziphi Spinosae, Fructus Gardeniae previous study (6) also showed that extracts obtained from SZS and FG have a synergistic effect on sedative hypnosis. However, the effect of SZS and FG on learning and memory has not been examined yet.

In the present study, we investigated the effects of the extracts of SZS and FG on the learning and memory of a mouse model using the step-through and -down passive avoidance tasks and Morris water maze tasks. The results showed that in both of the tasks, SZS and FG have a synergistic effect on improving the learning and memory of mice.

\section{Materials and methods}

Materials. SZS and FG oils were prepared by the Pharmacon Institute of FMMU using the supercritical $\mathrm{CO}_{2}$ extraction method. The compound oil of SZS and FG contains each herb at a 2:1 ratio. Scopolamine hydrobromide, cycloheximide, sodium nitrite and ethanol were purchased from Xuzhou Laien Pharmaceutical Co., Ltd. (Xuzhou, China), SigmaAldrich, Shanghai Zhizheng Chemical Engineering Co., Ltd. (Shanghai, China) and Xi'an Chemical Reagent Factory (Xi'an, Shanxi, China), respectively. Step-through and -down apparatuses were made by the Equipment Division of the Medical College of the Xi'an Jiaotong University, China. Morris water maze was constructed by the Chengdu TME Technology Co., Ltd. Female Kunming (KM) mice, clean (CL) animals ( $n=70)$, weighing 18-22 g, were obtained from the Animal Center of the Medical College of the Xian Jiaotong University.

Animal grouping and drug administration. Four experiments were designed to assess the effect of SZS and FG oils on improving murine learning and memory. In each experiment, the mice were randomized into seven groups $(n=10)$ : the saline control group, the learning and memory-impaired model group, the compound oil high-dose group, the compound oil medium-dose group, the compound oil low-dose group, the SZS oil only group and the FG oil only group. At 9:00 a.m. each day, for 15 days, the mice in each group were administered the following intragastrically: the saline control and the learning and memory-impaired model groups were administered normal saline; the compound oil high-, medium- and low-dose groups were administered 5.4, 2.7 and $1.35 \mathrm{~g} / \mathrm{kg}$ of compound oil of SZS and FG, respectively; while the SZS oil 
only and the FG oil only groups were given 1.8 and $0.9 \mathrm{~g} / \mathrm{kg}$ of SZS and FG oils, respectively.

Step-through and-down passive avoidance (PA) test in memory acquisition-impaired mice model. On day 16 of each experiment, the 9:00 am administration was carried out in the same manner for all the animal groups as previously described. One hour after administration, the animals in this experiment were injected with $1.5 \mathrm{mg} / \mathrm{kg}$ of scopolamine hydrobromide solution intraperitoneally in order to induce memory-acquisition impairment, with the exception of the saline control group. Ten minutes after injection, the mice were trained for step-through and -down passive avoidance task. In brief, for the step-through passive avoidance training, the mouse was placed into the illuminated compartment of the mouse step-through apparatus, facing away from the closed door connecting the illuminated and the dark compartments. Ten seconds later, the door connecting the 2 compartments was opened. The mouse would enter the dark compartment through the door due to its natural inclination. When its 4 paws touched the grid on the floor of the dark compartment, the door was closed and a brief electric foot shock $(0.4 \mathrm{~mA}$ for 2 sec) was applied. The mouse was kept in the dark compartment for $10 \mathrm{sec}$, and was then returned into its home cage. For the step-down training, the mouse was gently placed onto the platform of the step-down apparatus. When it stepped down and its 4 paws touched the grid on the floor of the apparatus, an electric foot shock $(0.4 \mathrm{~mA}$ for $5 \mathrm{sec})$ was applied. The mouse was then returned to its cage. The same training was repeated $1 \mathrm{~h}$ later. This time, however, the latency period for the mouse to step down the platform was measured. If the latency period was $>60 \mathrm{sec}$, i.e., if the mouse stayed on the platform for a period of $>60 \mathrm{sec}$ without stepping down, the mouse was returned to its home cage given that it had already memorized the unpleasant stimulus of stepping down onto the floor and learned to avoid it. If the latency period was $<60 \mathrm{sec}$, the training was repeated once more $1 \mathrm{~h}$ later. If the latency period remained $<60 \mathrm{sec}$, the mouse was excluded from the experiment.

Assessment of memory acquisition was conducted $24 \mathrm{~h}$ after the training session. For the step-through passive avoidance task, the latency period for the mouse to enter the dark compartment (with all four feet) and the number of times that the mouse entered the dark compartment within $5 \mathrm{~min}$ (no. of errors) were recorded. If the mouse did not enter the dark compartment in $5 \mathrm{~min}$, the assessment was ended, the step-through latency was recorded as $300 \mathrm{sec}$ and the number of errors was recorded as zero. For the step-down passive avoidance task, the latency period for the mouse to step down the platform and the number of times that the mouse stepped down the platform within 3 min (no. of errors) were recorded. If the mouse did not step down in $3 \mathrm{~min}$, the assessment was ended, the step-down latency was recorded as $180 \mathrm{sec}$ and the number of errors was recorded as zero.

Step-through and-down passive avoidance test with memory consolidation-impaired mice model. On day $16,1 \mathrm{~h}$ after the 9:00 a.m. administration, the mice included in this experiment were injected with $120 \mathrm{mg} / \mathrm{kg}$ cycloheximide solution intraperitoneally to induce memory consolidation impairment, with the exception of the saline control group. Ten minutes after the injection, the mice were trained for the step-through and -down passive avoidance task, as described above. Assessment of memory consolidation with step-through and -down passive avoidance task was conducted $24 \mathrm{~h}$ after the training session and was performed in the same manner.

Step-through and-down passive avoidance test with memory retrieval-impaired mice model. On day $16,1 \mathrm{~h}$ after the 9:00 a.m. administration, the mice in this experiment were trained for step-through and -down passive avoidance task, as described above. Twenty four hours after the training session, the mice were intragastrically administered $10 \mathrm{ml} /$ $\mathrm{kg}$ of ethanol to induce memory retrieval impairment, with the exception of the saline control group. Thirty minutes after the administration, assessment of memory retrieval with step-through and -down passive avoidance task was conducted and performed as described previously.

Morris water maze test of spatial learning and memory. From day 16 through 19, $1 \mathrm{~h}$ after the 9:00 a.m. procedural administration, the mice in this experiment were trained for the Morris water maze task. The water tub was 1.2 meters in diameter and 0.50 meters in height, and the inside wall was painted white. The tub was filled with water $\left(\sim 24^{\circ} \mathrm{C}\right)$ to a depth of 0.30 meters and was rendered opaque by adding white plastic foam. The mice were made visible by smearing picric acid (yellow color) on their fur. The pool area was arbitrarily divided into 4 quadrants: NE, SE, NW and SW. A transparent circular platform $(10 \mathrm{~cm}$ diameter) was arbitrarily placed in the center of the NW quadrant, $1 \mathrm{~cm}$ below the water level. The position of the hidden platform was kept constant throughout the training session. A digital camera connected to a computer recording system was mounted over the pool. Several colored shapes were placed around the pool and hung or pasted around the walls of the testing room as visual cues. Their positions remained unchanged during the entire experiment. Each mouse was subjected to 4 training trials each day (with a 5-min break between trials) for 4 consecutive days. On each trial the mouse was gently released into the water at a different location facing the wall of the pool. The time it spent to find the hidden platform (escape latency) was recorded. When the animal had found the platform, it was allowed to stay on the platform for 15 $\mathrm{sec}$ and was then removed from the maze. If the mouse failed to locate the platform in $60 \mathrm{sec}$, it was gently guided onto the platform and allowed to remain there for $15 \mathrm{sec}$. On day 19, following the last training trial, the mice were injected with $120 \mathrm{mg} / \mathrm{kg}$ sodium nitrite solution subcutaneously with a view to induce memory impairment, with the exception of the saline control group, which was injected with the same volume of normal saline.

On day 20, $1 \mathrm{~h}$ after the 9:00 a.m. procedural administration, the mice were assessed for the Morris water maze tasks (the position of the platform unchanged) and the escape latency was recorded as described previously. Subsequently, the transfer test (probe trial) was carried out in which the platform was removed from the pool, and the number of times each mouse crossed the previous platform location within $120 \mathrm{sec}$ was recorded. 

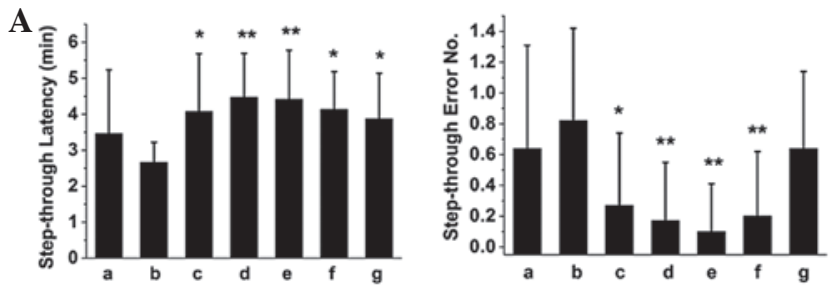

B
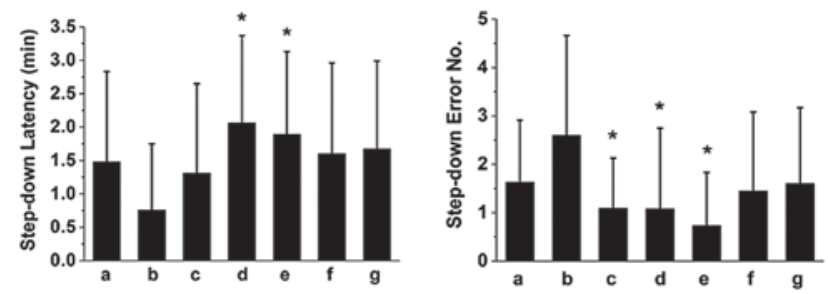

Figure 1. Effect of extracts of SZS and FG on the performance of mice with impairment in memory acquisition. Step-through and -down passive avoidance tasks were performed for mice with scopolamine treatment $(n=10$, mean $\pm \mathrm{SD}$ ). (A) Step-through latency and number of errors (Error no.) within 5 min was measured for the mouse groups. (B) Step-down latency and error no. within 3 min was measured for the mouse groups. a, normal saline; b, scopolamine; c, high-dose compound extracts of SZS and FG (CESF); d, medium-dose CESF; e, low-dose CESF; f, SZS extracts and g, FG extracts. ${ }^{*} \mathrm{P}<0.05 ;{ }^{* *} \mathrm{P}<0.01$ vs. the scopolamine group.
A

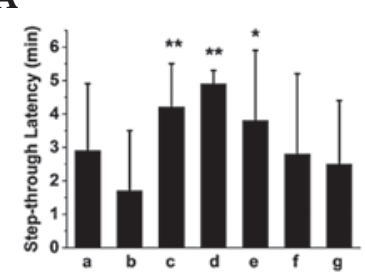

B

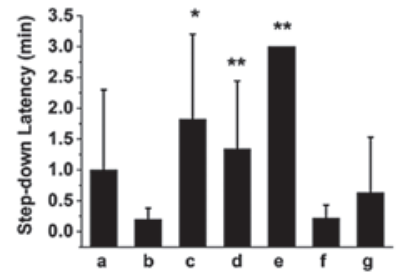

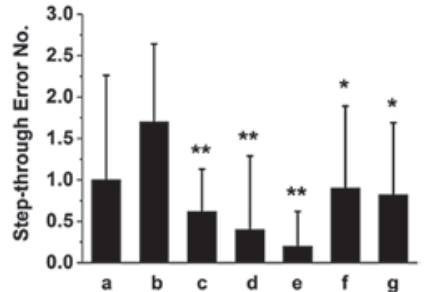

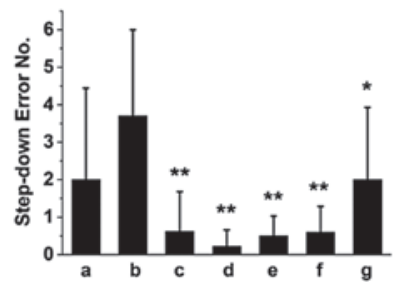

Figure 2. Effect of extracts of SZS and FG on the performance of mice with impairment in memory consolidation. Step-through and -down passive avoidance tasks were performed for mice with cycloheximide treatment $(n=10$, the mean $\pm S D)$. (A) Step-through latency and number of errors (Error no.) within 5 min was measured for the mouse groups. (B) Step-down latency and error no. within $3 \mathrm{~min}$ was measured for the mouse groups. a, normal saline; b, cycloheximide; c, high-dose compound extracts of SZS and FG (CESF) ; d, medium-dose CESF; e, low-dose CESF; f, SZS extracts and $\mathrm{g}$, FG extracts. ${ }^{*} \mathrm{P}<0.05 ;{ }^{* *} \mathrm{P}<0.01$ vs. the cycloheximide group.

\section{Results}

Extracts of SZS and FG synergistically improve learning and memory of mice in step-through and-down passive avoidance task. Scopolamine, cycloheximide and ethanol were used to induce impairment of memory acquisition, consolidation and
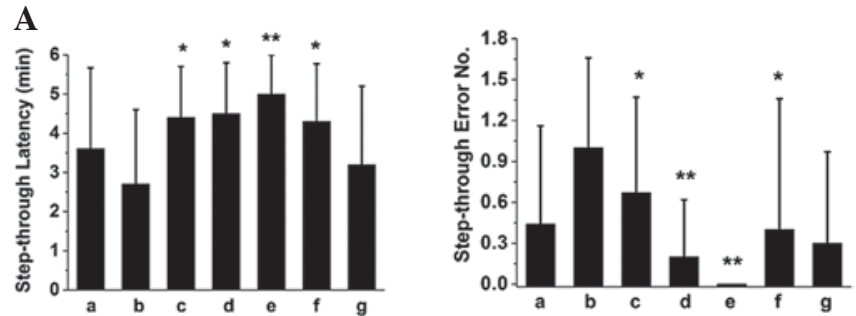

B
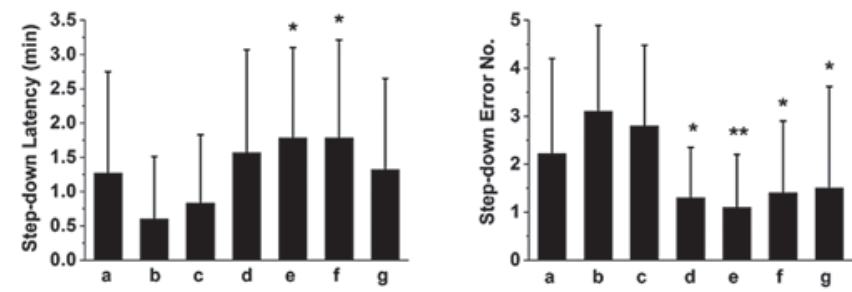

Figure 3. Effect of extracts of SZS and FG on the performance of mice with impairment in memory retrieval. Step-through and -down passive avoidance tasks were performed for mice with ethanol treatment $(n=10$, the mean $\pm S D)$. (A) Step-through latency and number of errors (Error no.) within 5 min was measured for the mouse groups. (B) Step-down latency and error no. within 3 min was measured for the mouse groups. a, normal saline; $b$, ethanol; $c$, high dose of compound extracts of SZS and FG (CESF); d, medium-dose CESF; e, low-dose CESF; f, SZS extracts and g, FG extracts. ${ }^{*} \mathrm{P}<0.05 ;{ }^{* *} \mathrm{P}<0.01$ vs. the ethanol group.

A
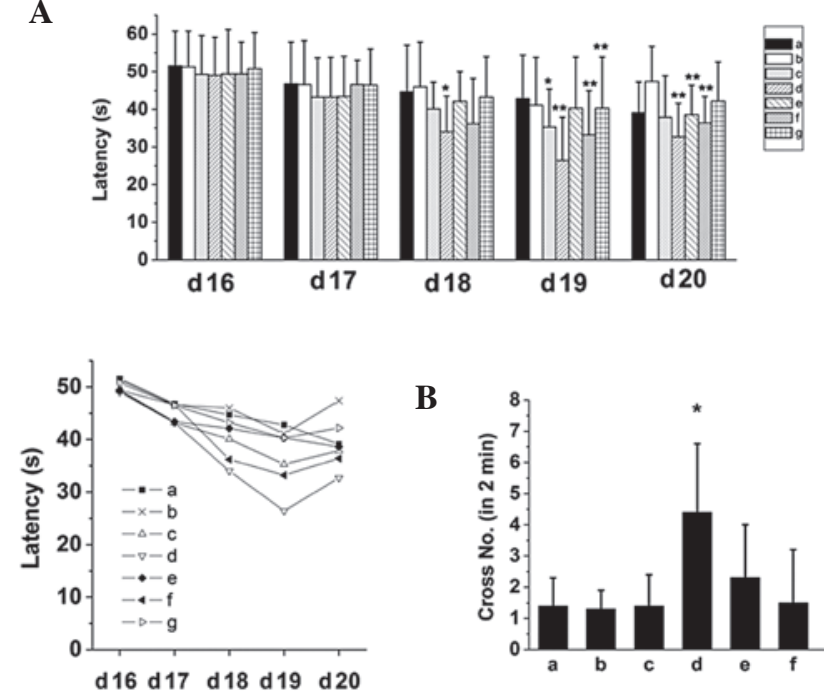

B

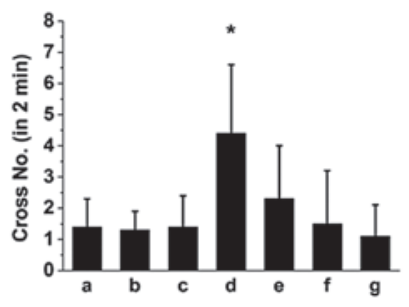

Figure 4. Effect of extracts of SZS and FG on mice with sodium nitrite induced memory impairment in Morris water maze task $(n=10$, the mean $\pm \mathrm{SD}$ ). The time the animal spent to find the hidden platform (escape latency) on (A) days 16-20 and the number of times the animal crosses the previous platform location within 2 min after the platform was removed from the pool (B) in the transfer test (cross no.) were recorded. a, normal saline; b, sodium nitrite; c, high dose of compound extracts of SZS and FG (CESF); d, medium-dose CESF; e, low-dose CESF; f, extracts of SZS and g, FG extracts. ${ }^{*} \mathrm{P}<0.05 ;{ }^{* *} \mathrm{P}<0.01$ vs. the normal saline group.

retrieval in mice, respectively. The results of step-through and -down passive avoidance tasks showed that SZS and FG oils improved the performance of mice with memory impairment in memory acquisition (Fig. 1), consolidation (Fig. 2) and retrieval (Fig. 3), since they were able to prolong the latency and reduce the error number. Notably, the compound oil of 
SZS and FG was the most effective, suggesting a synergistic effect for improving learning and memory in mice.

Effect of SZS and FG oils on the learning and memory of mice in the Morris water maze test. The results of the Morris water maze test demonstrated that the compound oil of SZS and FG improved the learning and memory in mice treated with sodium nitrite, a drug that induces memory retrieval-impairment, and the effects were the best at a medium, or $2.7 \mathrm{~g} / \mathrm{kg}$ dose (Fig. 4). Although SZS as well as FG oils improved the performance when used separately, the improvement was not markedly effective.

\section{Discussion}

SZS and FG were originally described in the Shennong Herbal (Shen Nong Ben Cao Jin), the oldest and the most classic Chinese materia medica book and have been used frequently by TCM doctors until present. SZS is classified as a tranquilizing herb that can tonify yin and nourish the heart. The indications of its clinical application include yin-blood deficiency and loss of nourishment of the heart and mind when manifested as emotional upset, insomnia and amnesia (4). FG is classified as a heat-clearing herb. It can purge fire and is especially good at clearing the heat of the heart and eliminating moody emotions. The indications of its clinical application include excess heat syndromes, such as depression, restlessness, irritability and delirium arising from heat suppressed in the heart (5). Although classified into different categories in TCM literature, based on their actions and properties, SZS and FG are usually used in combination to achieve better effects.

According to modern medical and pharmacological theories, the effective part of SZS and FG lies in the extracts (6). Our experiments demonstrated that the extracts of SZS and FG improved learning and memory in mice, however, SZS was more efficient compared to FG. Notably, SZS and FG have a synergistic effect, and the effect was the most prominent when the compound oil dose was medium ( $2.7 \mathrm{~g} / \mathrm{kg}$ body weight).

The effective ingredients or bioactive components of SZS and FG in improving learning and memory have not been specifically investigated. However, due to the composition of SZS and FG oils prepared with supercritical $\mathrm{CO}_{2}$, extraction is relatively simple and is rich in oleic and linoleic acids; as indicated by our previous studies (6-8), these two free fatty acids may therefore be candidate ingredients. Oleic acid can be acylated in vivo into oleamide and the latter has been reported to improve learning and memory in mice (9-11). Linoleic acid can be transformed in vivo into linolenic and further into arachidonic acid. Linolenic acid is able to increase membrane fluidity and plays an important role in neuron proliferation, nerve conduction and synapse formation (12-14). Arachidonic acid is one of the most abundant polyunsaturated fatty acids in the body and is essential for maintaining the physical property and function of the membrane (15-19). Arachidonic acid can also regulate hippocampal long-term potentiation (9). The metabolic product of arachidonic acid vasopressin may also improve learning and memory (20-22). Our study showed that SZS and FG oils have a synergistic effect on improving learning and memory. However, the mechanism of this synergy requires further investigation.

\section{References}

1. Yan ZH: Chinese Materia Medica (2nd edition). People's Medical Publishing House, Beijing, pp148-150, 2010.

2. Hou SL: Detailed annotation of eight hundred traditional Chinese medicines. Science and Technology Press of Henan, Zhengzhou, pp680-682, 2009.

3. Li J: Formulas of traditional Chinese medicine. Higher Education press, Beijing, pp233-234, 2006.

4. Zhang TM: Chinese Materia Medica. Higher Education press, Beijing, pp697-698, 2006.

5. Zhang TM: Chinese Materia Medica. Beijing: Higher Education press, pp380-382, 2006.

6. Li B, Xia C and Yuan B: Sedative and hypnotic effects of oil of spine date seeds from different extraction technologies in mice. J Xian Jiaotong Univ Med Sci 29: 227-229, 2008 (In Chinese)

7. Zhang Z, Li B, Chen Y, et al: Assay of the compositions of the compound oil of semen Ziziphi spinosae and fructus Gardeniae. J Shanxi Med Univ 38: 792-793, 2009.

8. Li B, Chen Y, Zhang Z, et al: Effect of compound jujube seed oil and gardenia oil on improving the ability of learning and memory in mice. J Xian Jiaotong Univ Med Sci 31: 673-676, 707, 2010

9. Akanmu MA, Adeosun SO and Ilesanmi OR: Neuropharmacological effects of oleamide in male and female mice. Behav Brain Res 182: 88-94, 2007.

10. Micale V, Cristino L, Tamburella A, et al: Enhanced cognitive performance of dopamine D3 receptor 'knock-out' mice in the step-through passive-avoidance test: assessing the role of the endocannabinoid/endovanilloid systems. Pharmacol Res 61: 531-536, 2010.

11. Ahmadi S, Malekmohammadi $\mathrm{N}$ and Zarrindast MR: Repeated histamine pretreatment decreased amnesia induced by post-training administration of the drug in a step-down inhibitory avoidance test in mice. Arch Iran Med 13: 209-216, 2010.

12. Yang X, Sheng W, Sun GY, et al: Effects of fatty acid unsaturation numbers on membrane fluidity and $\alpha$-secretase-dependent amyloid precursor protein processing. Neurochem Int 58: 321-329, 2011.

13. Saha SS, Chakraborty A, Ghosh S, et al: Comparative study of hypocholesterolemic and hypolipidemic effects of conjugated linolenic acid isomers against induced biochemical perturbations and aberration in erythrocyte membrane fluidity. Eur J Nutr 51: 483-495, 2012.

14. Lauretani F, Bandinelli S, Bartali B, et al: Omega-6 and omega-3 fatty acids predict accelerated decline of peripheral nerve function in older persons. Eur J Neurol 14: 801-808, 2007.

15. Villegas-Comonfort S, Serna-Marquez N, GalindoHernandez $\mathrm{O}$, et al: Arachidonic acid induces an increase of $\beta$-1,4-galactosyltransferase I expression in MDA-MB-231 breast cancer cells. J Cell Biochem 113: 3330-3341, 2012.

16. Rossen NS, Hansen AJ, Selhuber-Unkel C, et al: Arachidonic acid randomizes endothelial cell motion and regulates adhesion and migration. PLoS One 6: e25196, 2011.

17. Das UN: Long-chain polyunsaturated fatty acids in growth and development of brain and memory. Nutrition 19: 62-65, 2003.

18. Fu ZY, Wang YP and Chen Y: Observation of insulin exocytosis by a pancreatic $\beta$ cell line with total internal reflection fluorescence microscopy. Chin Med Sci J 26: 60-63, 2011.

19. Matsuyama M and Yoshimura R: Arachidonic acid pathway: A molecular target in human testicular cancer (Review). Mol Med Rep 2: 527-531, 2009.

20. DeCostanzo AJ, Voloshyna I, Rosen ZB, et al: 12-Lipoxygenase regulates hippocampal long-term potentiation by modulating L-type $\mathrm{Ca}^{2+}$ channels. J Neurosci 30: 1822-1831, 2010.

21. Alescio-Lautier B and Soumireu-Mourat B: Role of vasopressin in learning and memory in the hippocampus. Prog Brain Res 119: 501-521, 1998.

22. Engelmann $\mathbf{M}$ and Wotjak CT, Ebner $\mathrm{K}$ and Landgraf R: Behavioral impact of intraseptally released vasopressin and oxytocin in rats. Exp Physiol 85: S125-S130, 2000. 